\title{
Differential expression of NR3C2 in cancers of the breast.
}

Shahan Mamoor, MS ${ }^{1}$

$3 \quad{ }^{1}$ shahanmamoor@gmail.com

East Islip, NY 11730

Breast cancer affects women at relatively high frequency ${ }^{1}$. We mined published microarray datasets ${ }^{2,3}$ to determine in an unbiased fashion and at the systems level genes most differentially expressed in the primary tumors of patients with breast cancer. We report here significant differential expression of the gene encoding nuclear receptor subfamily 3 group $\mathrm{C}$ member 2, NR3C2, when comparing primary tumors of the breast to the tissue of origin, the normal breast. NR3C2 mRNA was present at significantly lower quantities in tumors of the breast as compared to normal breast tissue. Analysis of human survival data revealed that expression of $\mathrm{NR} 3 \mathrm{C} 2$ in primary tumors of the breast was correlated with distant metastasis-free survival in patients with luminal A subtype cancers, demonstrating a relationship between primary tumor expression of a differentially expressed gene and patient survival outcomes influenced by molecular subtype. NR3C2 may be of relevance to initiation, maintenance or progression of cancers of the female breast.

Keywords: breast cancer, NR3C2, nuclear receptor subfamily 3 group $\mathrm{C}$ member 2, systems biology of breast cancer, targeted therapeutics in breast cancer. 
Invasive breast cancer is diagnosed in over a quarter of a million women in the United States each year $^{1}$ and in 2018 , breast cancer was the leading cause of cancer death in women worldwide ${ }^{4}$. While patients with localized breast cancer are provided a 99\% 5-year survival rate, patients with regional breast cancer, cancer that has spread to lymph nodes or nearby structures, are provided an $86 \% 5$-year survival rate $^{5,6}$. Patients with metastasis to distant sites, like the brain, are provided a $27 \% 5$-year survival rate ${ }^{5,6}$. Understanding how primary tumors are most transcriptionally different from the tissue from which they originate, the breast, can facilitate development of novel diagnostic and therapeutics to promote early detection and enhanced treatment, and contribute to efforts to prevent progression to metastatic stages. We mined published microarray data ${ }^{2,3}$ to understand at the transcriptome level and in an unbiased fashion genes most differentially expressed in primary tumors of the breast as compared to normal breast tissue. nuclear receptor subfamily 3 group $\mathrm{C}$ member 2 emerged as among the most differentially expressed genes in cancer of the female breast.

\section{Methods}

We utilized datasets GSE109169² and GSE42568 ${ }^{3}$ for this differential gene expression analysis of female breast cancer. GSE109169 was generated using Affymetrix Human Exon 1.0 ST Array technology with $n=25$ normal breast tissue and $n=25$ tumors of the breast; analysis was performed using platform GPL5175. The tissues from this first dataset are paired tissues ( 25 tumors matching 25 breast tissues from 25 patients). GSE42568 was generated using Affymetrix Human Genome U133 Plus 2.0 array technology with $n=17$ normal breast tissue biopsies and $n=104$ primary breast tumor biopsies from patients with breast cancer; analysis was performed using platform GPL570; the majority of patients whose tumors were analyzed were age 50 or older. The Benjamini and Hochberg method of p-value adjustment was used for ranking of differential expression but raw $p$-values were used to assess statistical significance of global differential expression. Log-transformation of data was auto-detected, and the NCBI generated category of platform annotation was used. A statistical test was performed to evaluate whether NR3C2 expression was significantly different between primary breast tumors and breast tissue using a two-tailed t-test. For Kaplan-Meier survival analysis, we used the Kaplan-Meier plotter online tool ${ }^{7}$ for correlation of NR3C2 mRNA expression levels with distant metastasis-free survival (DMFS) in $n=571$ patients with basal subtype cancer, $n=1260$ patients with luminal A subtype cancer, $n=756$ patients with luminal B subtype cancer, and $n=178$ patients with HER2+ cancer.

\section{Results}

\section{NR3C2 is differentially expressed in primary tumors of the breast.}

Comparison of 25 normal breast tissues to 25 tumors of the breast ${ }^{2}$ from patients with early-onset breast cancer revealed that the gene encoding the nuclear receptor subfamily 3 group $\mathrm{C}$ member 2 , NR3C2, was among the genes most differentially expressed in tumors of the breast in human breast cancer (Chart 1). When sorting each of the genes expressed in tumors of the breast based on significance of difference as compared to normal breast tissue, NR3C2 ranked 31 out of 19076 total transcripts, equating to $99.8 \%$ differential expression (Chart 1). Differential expression of NR3C2 in female breast cancer was statistically significant (Chart $1 ; p=6.84 \mathrm{E}-18$ ).

Analysis of a second microarray dataset ${ }^{3}$, here in 104 tumors from patients aged 31 to 89 at diagnosis, the majority of which were above age 50, again revealed significant differential expression of NR3C2 in human breast cancer (Chart 2). When sorting each of the genes expressed in the tumors of 
patients with breast cancer based on significance of difference as compared to normal breast tissue, NR3C2 ranked 2523 out of 54675 total transcripts, equating to $95.4 \%$ differential expression (Chart 2). Differential expression of NR3C2 in the tumors of patients with breast cancer was statistically significant (Chart $2 ; p=8.32 \mathrm{E}-11$ ). These data suggested that differential expression of NR3C2 was not an artifact of a single microarray dataset, nor was it strictly associated with early-onset breast cancer, rather a general feature of cancers of the breast.

NR3C2 is expressed at significantly lower levels in breast tumors as compared to the breast.

We obtained exact mRNA expression levels for NR3C2 from the breast and from breast tumors to understand the magnitude and direction of NR3C2 expression change. NR3C2 was expressed at lower levels in tumors of the breast as compared to normal breast tissue (Figure 1). Decreased expression of NR3C2 in primary breast tumors was statistically significant (Figure 1: $p<0.0001$ ). NR3C2 was expressed at $7.39 \pm 0.25$ arbitrary units (AU) in normal breast tissue, while it was expressed at $6.28 \pm 0.35 \mathrm{AU}$ in tumors of the breast. We calculated a mean fold change of 0.85 in NR3C2 mRNA levels when comparing primary tumors of the breast to normal breast tissues.

\section{NR3C2 expression correlates with survival outcomes in luminal A subtype human breast cancer.}

We performed Kaplan-Meier survival analysis in an attempt to correlate NR3C2 mRNA expression levels with survival outcomes in patients with breast cancer, evaluating correlations between molecular subtype and distant metastasis-free survival. We observed a statistically significant correlation between NR3C2 expression and distant metastasis-free survival (DMFS) in patients with luminal A subtype breast cancer (Figure 2; log rank $p$-value: 0.027 for distant metastasis-free survival, hazard ratio: 0.75 (0.57-0.97) (Fig. 2)). NR3C2 mRNA levels were a positive prognostic indicator in luminal A subtype breast cancer patients. Median DMFS was 130.72 months for luminal A patients with low tumor expression of NR3C2 while median DMFS was 149 months for luminal A patients with high tumor expression of NR3C2 (Chart 3). NR3C2 primary tumor expression was not correlated with distant metastasis-free survival in basal subtype (Figure 2; log rank $p$-value: 0.56 for DMFS, hazard ratio: 0.91 (0.67-1.24) (Fig. 2), luminal B subtype (Figure 2; log rank $p$-value: 0.39 for DMFS, hazard ratio: 1.13 (0.86-1.5) (Fig. 2), or in HER2+ breast cancer (Figure 2; log rank $p$-value: 0.43 for DMFS, hazard ratio: $1.22(0.74-2.01)$ (Fig. 2)).

Thus, through comparative transcriptome analysis of primary tumors of the breast and normal breast tissue, we found that differential expression and down-regulation of NR3C2 was among the most significant transcriptional features in primary tumors from patients with breast cancer. NR3C2 expression in primary tumors of the breast was correlated with distant metastasis-free survival in patients with luminal A disease, with mRNA levels of NR3C2 a positive prognostic indicator for luminal A subtype breast cancer patients.

\section{Discussion}

Invasive breast cancer is a medical problem with a $27 \% 5$-year survival rate for women whose disease has spread to distant sites ${ }^{5,6}$. To facilitate understanding of the basic transcriptional differences between primary tumors of the breast and the tissues from which these tumors originate, normal breast tissues, we performed comparative transcriptome analysis using two independently published microarray datasets $^{2,3}$, providing evidence here that differential expression of nuclear receptor subfamily 3 group C member 2, encoded by NR3C2, is a defining transcriptional feature of human breast cancer: in early onset-breast cancer, and in patients diagnosed after age 50. NR3C2 was expressed at significantly lower levels in primary tumors from patients with breast cancer as compared to normal breast tissue. Importantly, in patients with luminal A breast cancers, expression of NR3C2 was correlated with distant 


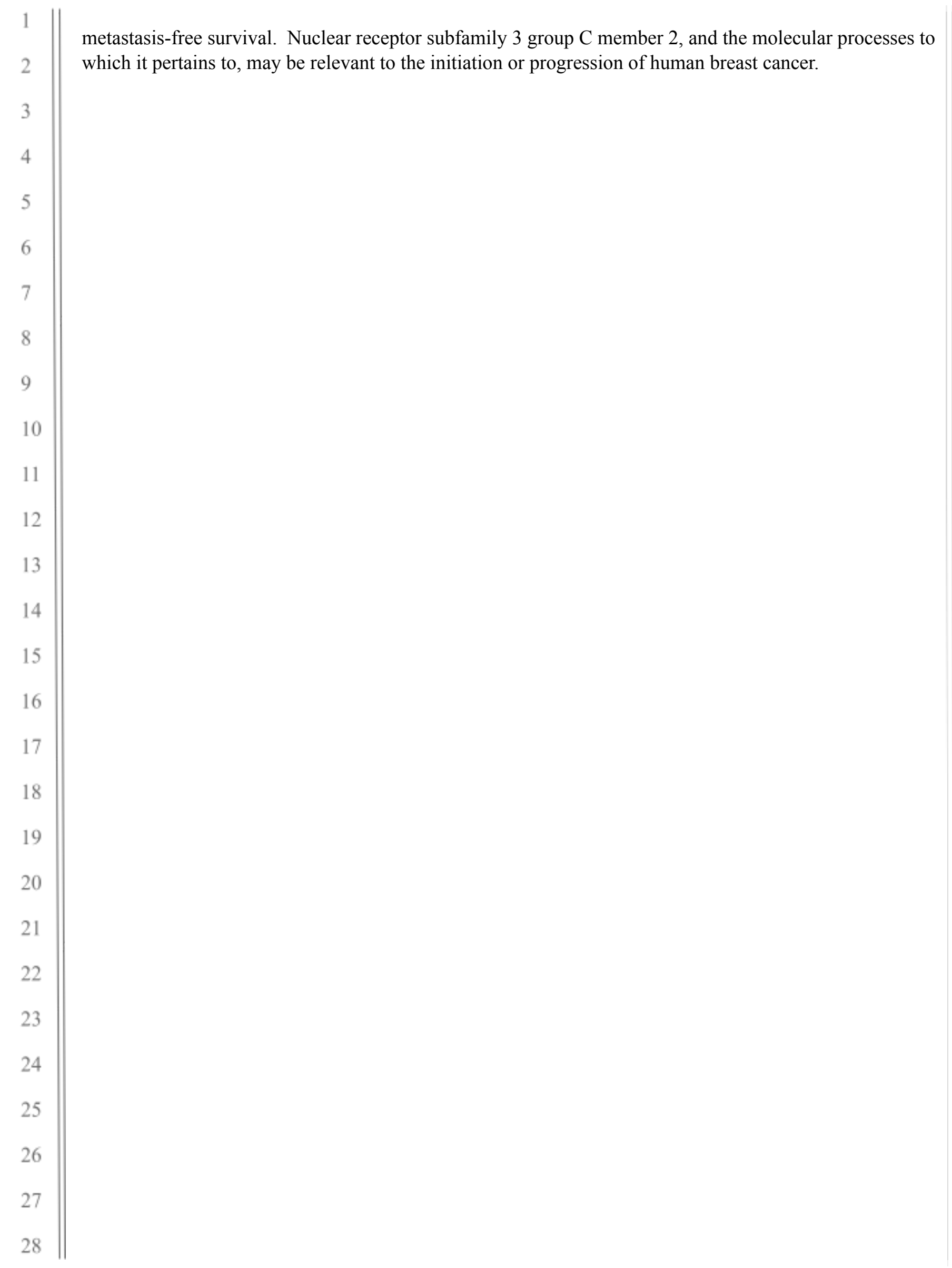




\section{References}

1. DeSantis, C.E., Ma, J., Goding Sauer, A., Newman, L.A. and Jemal, A., 2017. Breast cancer statistics, 2017, racial disparity in mortality by state. CA: a cancer journal for clinicians, 67(6), pp.439-448.

2. Chang, J.W., Kuo, W.H., Lin, C.M., Chen, W.L., Chan, S.H., Chiu, M.F., Chang, I.S., Jiang, S.S., Tsai, F.Y., Chen, C.H. and Huang, P.H., 2018. Wild-type p53 upregulates an early onset breast cancer-associated gene GAS7 to suppress metastasis via GAS7-CYFIP1- mediated signaling pathway. Oncogene, 37(30), pp.4137-4150.

3. Clarke, C., Madden, S.F., Doolan, P., Aherne, S.T., Joyce, H., O’driscoll, L., Gallagher, W.M., Hennessy, B.T., Moriarty, M., Crown, J. and Kennedy, S., 2013. Correlating transcriptional networks to breast cancer survival: a large-scale coexpression analysis. Carcinogenesis, 34(10), pp.2300-2308.

4. Bray, F., Ferlay, J., Soerjomataram, I., Siegel, R.L., Torre, L.A. and Jemal, A., 2018. Global cancer statistics 2018: GLOBOCAN estimates of incidence and mortality worldwide for 36 cancers in 185 countries. CA: a cancer journal for clinicians, 68(6), pp.394-424.

5. ACS Cancer Facts \& Figures 2019.

https://www.cancer.net/cancer-types/breast-cancermetastatic/statistics.

6. Survival Rates for Breast Cancer. https://www.cancer.org/cancer/breast-cancer/ understanding-a-breast-cancer-diagnosis/breast-cancer-survival-rates.html

7. Györffy, B., Lanczky, A., Eklund, A.C., Denkert, C., Budczies, J., Li, Q. and Szallasi, Z., 2010. An online survival analysis tool to rapidly assess the effect of 22,277 genes on breast cancer prognosis using microarray data of 1,809 patients. Breast cancer research and treatment, 123(3), pp.725-731. 


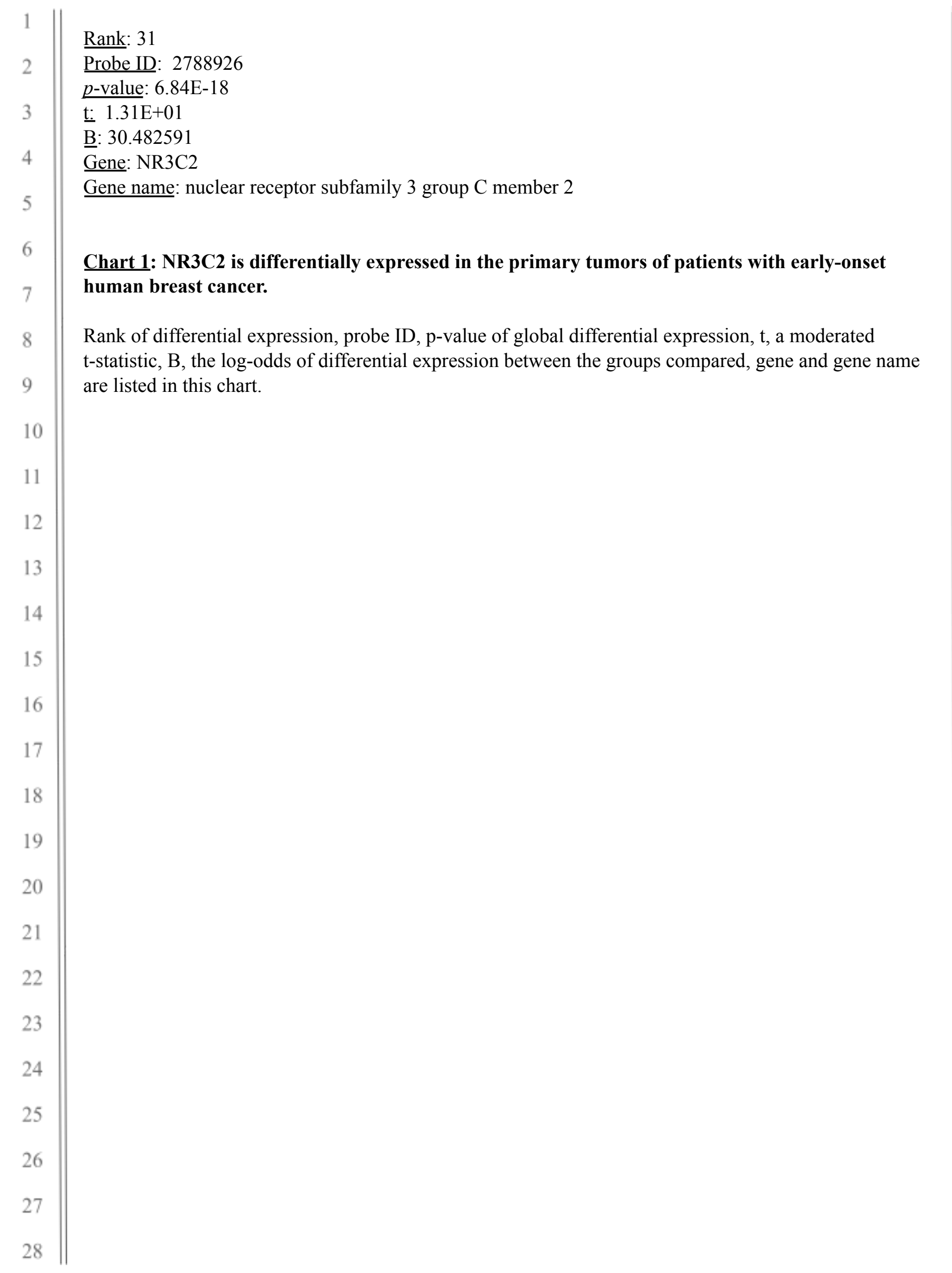




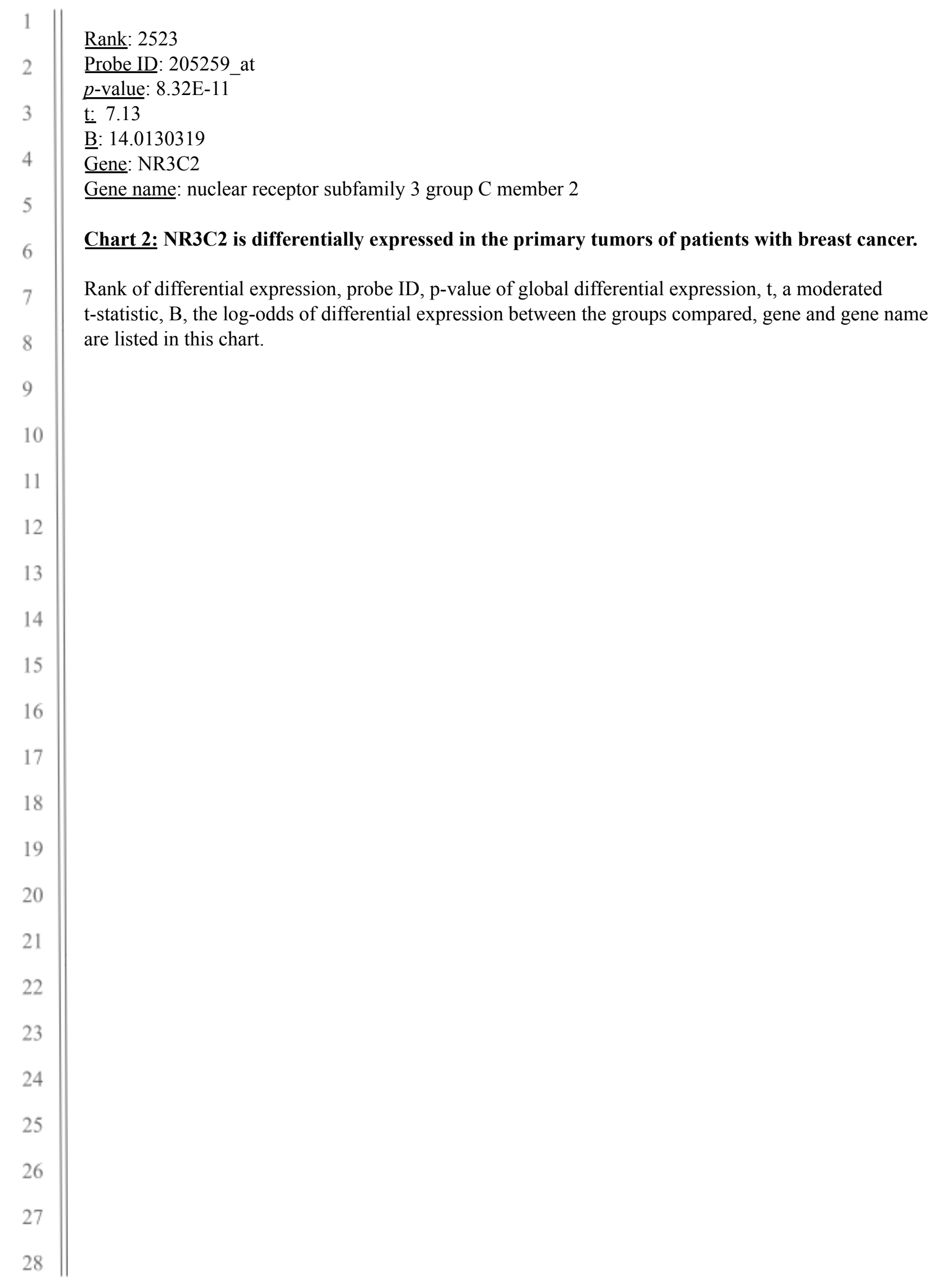




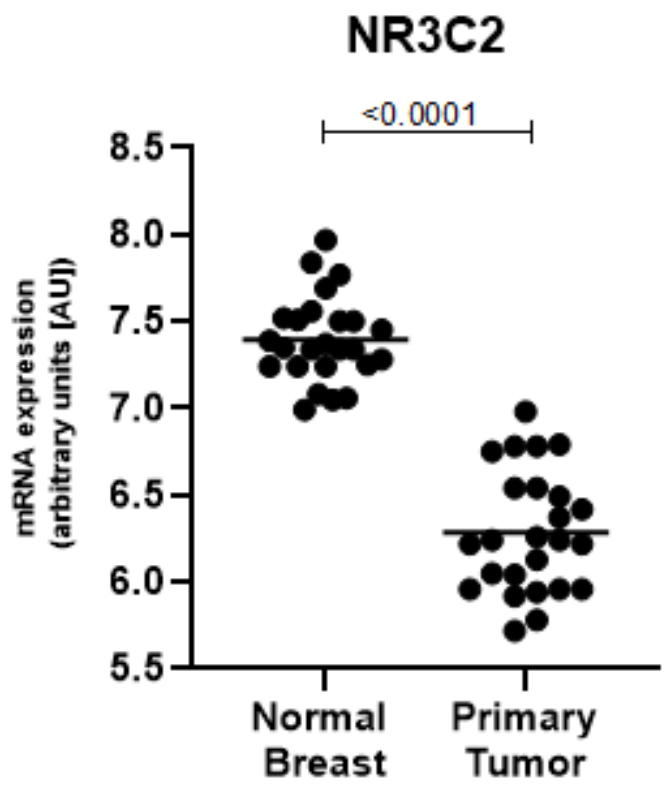

Figure 1: NR3C2 is expressed at significantly higher levels in primary breast tumors as compared to normal breast tissue.

The mRNA expression level of NR3C2 in normal breast tissue (left) and in primary tumors of the breast (right) is graphically depicted with the result of a statistical test evaluating significance of difference in NR3C2 expression between normal breast tissue and primary tumors of the breast, a $p$-value, is $p<0.0001$. 

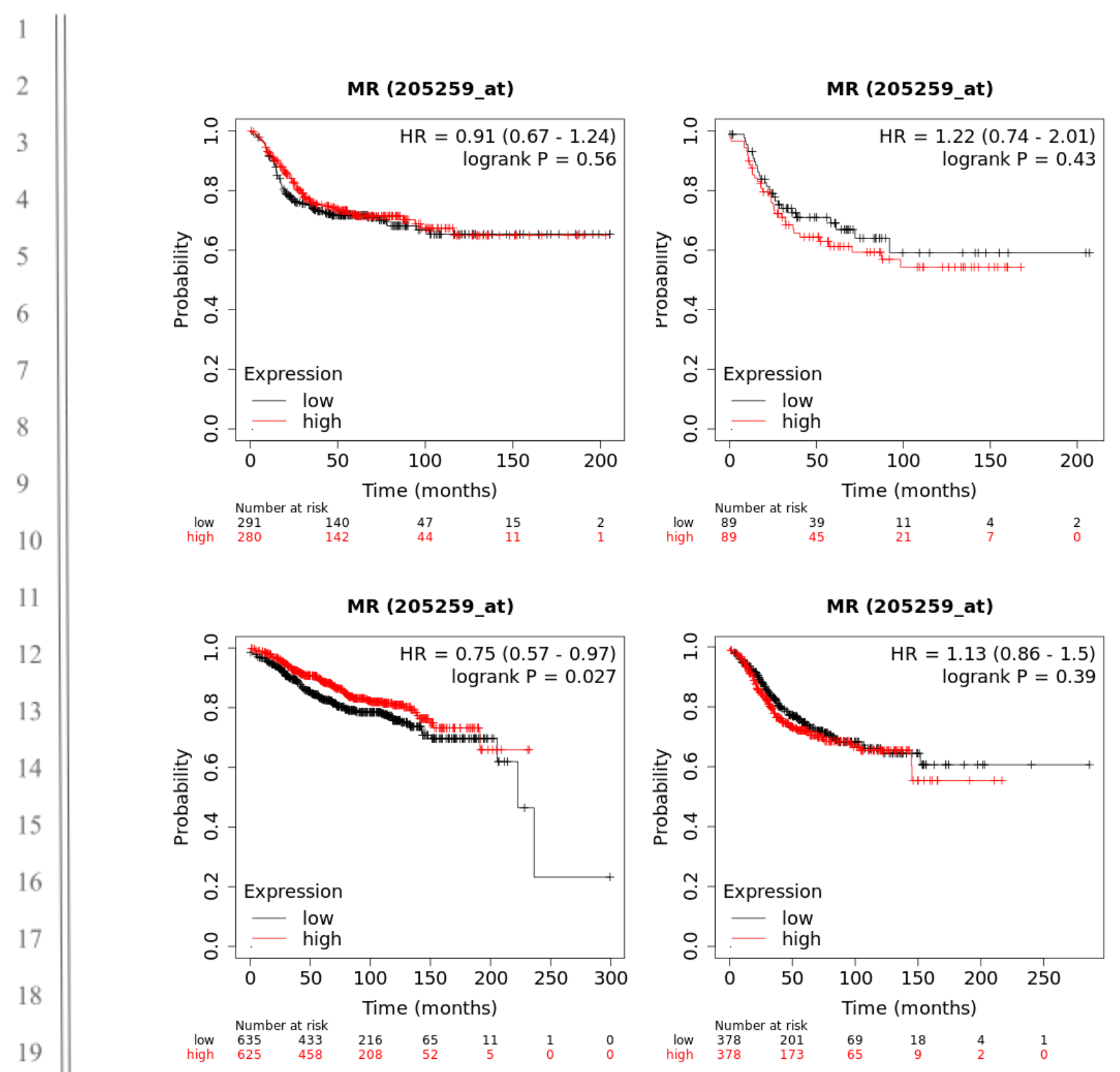

Figure 2: NR3C2 expression correlates with distant metastasis-free survival in patients with luminal A breast cancer, but not in patients with basal subtype, luminal B, or HER2+ cancer.

Depicted in this Kaplan-Meier plot is the probability of distant metastasis-free survival (DMFS) for $n=571$ patients with basal subtype breast cancer (upper left), $n=178$ patients with HER2+ breast cancer (upper right), $n=1260$ patients with luminal A breast cancer (lower left), and $n=756$ patients with luminal B breast cancer (lower right), stratified into two groups, based on low or high expression of NR3C2 in patient primary tumors. The log rank p-value denoting statistical significance of difference in distant metastasis-free survival when comparing the two groups, as well as hazard ratio for this comparison is listed above. Listed below is the number of patients at risk (number of patients alive) per interval, after stratification based on NR3C2 expression; in the first interval, number at risk is number of patients alive; in each subsequent interval, number at risk is the number at risk less those who have expired or are censored. 


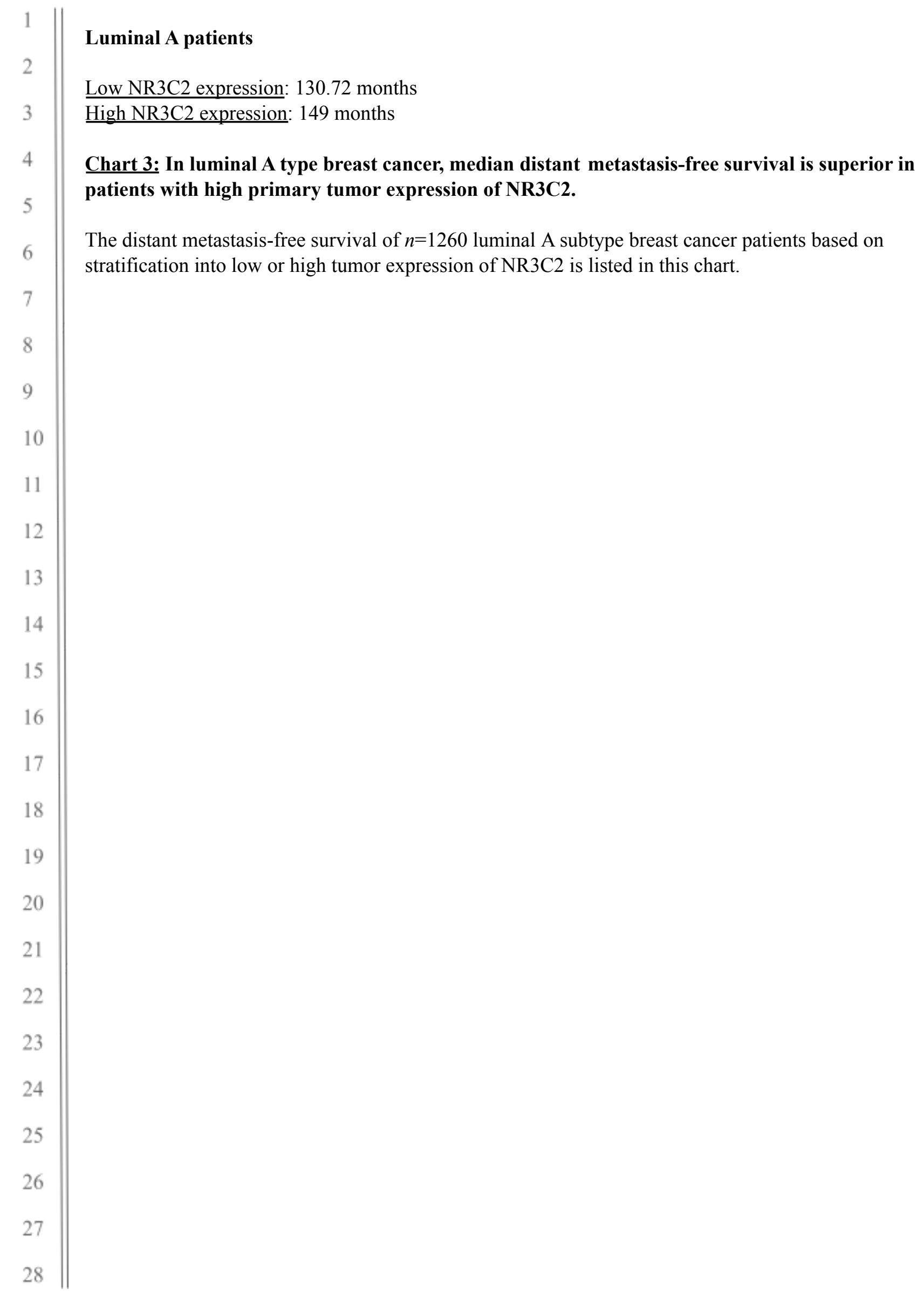

